\title{
A Controlled Trial to Determine the Efficacy of Red and Near-Infrared Light Treatment in Patient Satisfaction, Reduction of Fine Lines, Wrinkles, Skin Roughness, and Intradermal Collagen Density Increase
}

\author{
Alexander Wunsch ${ }^{1}$ and Karsten Matuschka ${ }^{2}$
}

\begin{abstract}
Objective: The purpose of this study was to investigate the safety and efficacy of two novel light sources for large area and full body application, providing polychromatic, non-thermal photobiomodulation (PBM) for improving skin feeling and appearance. Background data: For non-thermal photorejuvenation, laser and LED light sources have been demonstrated to be safe and effective. However, lasers and LEDs may offer some disadvantages because of dot-shaped (punctiform) emission characteristics and their narrow spectral bandwidths. Because the action spectra for tissue regeneration and repair consist of more than one wavelength, we investigated if it is favorable to apply a polychromatic spectrum covering a broader spectral region for skin rejuvenation and repair. Materials and methods: A total of 136 volunteers participated in this prospective, randomized, and controlled study. Of these volunteers, 113 subjects randomly assigned into four treatment groups were treated twice a week with either $611-650$ or $570-850 \mathrm{~nm}$ polychromatic light (normalized to $\sim 9 \mathrm{~J} / \mathrm{cm}^{2}$ in the range of $\left.611-650 \mathrm{~nm}\right)$ and were compared with controls $(n=23)$. Irradiances and treatment durations varied in all treatment groups. The data collected at baseline and after 30 sessions included blinded evaluations of clinical photography, ultrasonographic collagen density measurements, computerized digital profilometry, and an assessment of patient satisfaction. Results: The treated subjects experienced significantly improved skin complexion and skin feeling, profilometrically assessed skin roughness, and ultrasonographically measured collagen density. The blinded clinical evaluation of photographs confirmed significant improvement in the intervention groups compared with the control. Conclusions: Broadband polychromatic PBM showed no advantage over the red-light-only spectrum. However, both novel light sources that have not been previously used for PBM have demonstrated efficacy and safety for skin rejuvenation and intradermal collagen increase when compared with controls.
\end{abstract}

\section{Introduction}

A LTERING CELLULAR FUNCTION USING LOW LEVEL, nONthermal LED light is called photobiomodulation (PBM) or low-level light therapy (LLLT), and is a medical treatment modality of increasing clinical importance. ${ }^{1}$ Because of the combination of high degree of penetration in skin $^{2}$ and absorption by respiratory chain components, light in the spectral range from 600 to $1300 \mathrm{~nm}$ is useful for promoting wound healing, tissue repair, and skin rejuvenation. ${ }^{3-5}$ In contrast to traumatic ablative (e.g., laser resurfacing) and non-ablative (e.g., intense pulsed light [IPL]) skin rejuvenation modalities that induce secondary tissue repair by causing controlled damage to either the epidermis or the dermis, PBM is atraumatic, and bypasses the initial destructive step by directly stimulating regenerative processes in the skin. Its action mechanisms encompass increased cellular proliferation, migration, and adhesion. ${ }^{6}$ Important cell types for skin and tissue regeneration are fibroblasts, keratinocytes, and immune cells (mast cells, neutrophils, and macrophages), which can be stimulated using specific wavelengths with significant tissue penetration properties. ${ }^{7}$ The known severe side effects of traumatic skin rejuvenation procedures, such as inflammation, unpleasant pain perception, and prolonged social down time, ${ }^{8}$ are unknown in PBM; PBM has been successfully administered to reduce common symptoms of laser resurfacing

\footnotetext{
${ }^{1}$ Medical Light Consulting, Heidelberg, Germany.
}

${ }^{2}$ JK-International GmbH, Windhagen, Germany. 
and IPL treatment. ${ }^{9}$ Photon emitters, such as lasers or LEDs, have proven to be effective light sources for PBM during recent decades, thereby demonstrating that it is not the technical type of light source but the treatment parameters such as wavelength, irradiance, and fluence that are likely to be accountable for the effects. ${ }^{10}$ However, laser and LED light sources may offer some disadvantages because of their dotshaped (punctiform) emission characteristics and narrow spectral bandwidths. Because the action spectra for tissue regeneration and repair consist of more than one wavelength, 7,11 it might be favorable to apply a polychromatic spectrum covering a broader spectral region for skin rejuvenation and skin repair. We investigated the safety and efficacy of a novel non-thermal, non-ablative, atraumatic, polychromatic low-level light treatment modality with a focus on pleasant skin feeling, improved skin appearance, intradermal collagen increase, and the visible reduction of fine lines and wrinkles in a prospective, randomized, controlled trial that consisted of 136 volunteers.

\section{Materials and Methods}

\section{Study population and design}

We conducted a randomized, controlled clinical trial between January 2012 and December 2012. Table 1 summarizes the baseline $(\mathrm{t} 0)$ characteristics of the subject groups.

The subjects were between 27 and 79 years of age. Inclusion criteria were the capacity to independently position oneself to use the device, the capacity to understand the treatment, a signed declaration of consent, and interest in continuous participation. The exclusion criteria were physical and psychological disease casting doubt on the capacity to consent, preliminary treatment with red light within the 6 months prior to the beginning of the study, recent invasive cosmetic procedures such as Botox during the 12 months prior to the beginning of the study, acute or prior skin cancer, acute skin disease requiring dermatological treatment, existing or planned pregnancy, lactation, history of photosensitivity or recent use of photosensitizing medication, epilepsy, and the tendency to faint. All of the participants gave written informed consent for this study, which was approved by the Ethics Committee of the Medical Association (Landesärztekammer) Baden-Württemberg, Stuttgart, Germany. The investigation was conducted in accordance with the Declaration of Helsinki (DoH/Oct2008). After the declaration of informed consent following examination of the inclusion and exclusion criteria, each participant was assigned to one of four groups using a computerized randomization process. Group 5 was mainly recruited from employees of the JK company without randomization, and served as the control. Groups 1-4 were treated twice a week with 30 treatments in total, starting in January 2012. To minimize the influence of seasonal changes, the time interval for data acquisition at the baseline, t15, t30, and follow-up examinations was restricted to 1 month. The data acquisition at baseline was completed in February 2012, and all of the volunteers finished treatment 30 (t30) in June 2012.

The control group did not receive any treatment, as the therapy cannot be blinded, and a sham light source without any effect most likely does not exist. The control group volunteers participated in the clinical measurements only, and the acquisition of subjective parameters such as skin feeling and skin complexion was not conducted. Because of the similar spectral lamp characteristics for groups 1 and 2 and groups 3 and 4, groups 1 and 2 were combined for evaluation as the "mid-pressure lamp group" [energizing light technology (ELT)], and groups 3 and 4 were evaluated together as the "low-pressure lamp group" [red light technology (RLT)] to obtain larger group sizes and, therefore, higher statistical power. Nevertheless, the subdivision into groups 1-4 allowed us to compare outcomes based on different treatment parameters, such as spectral distribution, irradiance, and fluence. A questionnaire concerning the tolerability of the application was filled in after each treatment (t1-t30). Digital photographs and clinical measurements were taken, and subjective questionnaires were used to assess complexion and skin feeling at the baseline $(\mathrm{t} 0)$ and after 15 (t15) and 30 treatments (t30). The follow-up acquisition of

Table 1. Baseline (t0) Characteristics of the Subject Groups

\begin{tabular}{|c|c|c|c|}
\hline & $R L T(\mathrm{n}=57)$ & $\operatorname{ELT}(\mathrm{n}=48)$ & Controls $(\mathrm{n}=23)$ \\
\hline \multicolumn{4}{|l|}{ Sex } \\
\hline Female & $49 / 86.0 \%$ & $34 / 70.8 \%$ & $15 / 65.2 \%$ \\
\hline Male & $8 / 14.0 \%$ & $14 / 29.2 \%$ & $8 / 34.8 \%$ \\
\hline $\operatorname{Age}^{\mathrm{a}}$ & $46.2 \pm 9.0$ & $48.6 \pm 9.8$ & $44.4 \pm 10.2$ \\
\hline Weight $^{\mathrm{a}}$ & $72.9 \pm 15.22$ & $73.4 \pm 13.7$ & $73.7 \pm 13.4$ \\
\hline Skin complexion (subjective) ${ }^{b}$ & $4.54 \pm 1.92$ & $4.87 \pm 2.02$ & \\
\hline Skin feeling (subjective) ${ }^{\mathrm{b}}$ & $5.33 \pm 2.04$ & $5.24 \pm 2.18$ & \\
\hline Skin roughness $\left(R_{a}\right)^{b}$ & $15.29 \pm 4.20$ & $14.84 \pm 4.04$ & $11.79 \pm 2.17$ \\
\hline Collagen intensity score ${ }^{c}$ & $20.40 \pm 6.55$ & $18.96 \pm 3.54$ & $23.22 \pm 7.36$ \\
\hline \multicolumn{4}{|l|}{ Expert wrinkle assessment ${ }^{\mathrm{d}}$} \\
\hline No/shallow or fine wrinkles & $14 / 24.6 \%$ & $17 / 35.4 \%$ & $5 / 21.7 \%$ \\
\hline Moderate wrinkles & $20 / 35.1 \%$ & $11 / 22.9 \%$ & $6 / 26.1 \%$ \\
\hline Prominent or deep wrinkles & $13 / 22.8 \%$ & $11 / 22.9 \%$ & $9 / 39.1 \%$ \\
\hline No majority vote possible & $10 / 17.5 \%$ & $9 / 18.8 \%$ & $3 / 13.0 \%$ \\
\hline
\end{tabular}

${ }^{\mathrm{a}}$ Values represent means $\pm \mathrm{SD}$ at $\mathrm{t} 0$.

${ }^{b}$ Values represent means $\pm S D$ at $t 0$; small numbers indicate good values.

${ }^{c}$ Values represent means $\pm \mathrm{SD}$ at $\mathrm{t} 0$; large numbers indicate good values.

${ }^{\mathrm{d}}$ Majority vote of three blinded expert reviewers, based on the Modified Fitzpatrick Wrinkle Scale.

RLT, red light technology; ELT, energizing light technology. 
Table 2. Characteristics of the Treatment Units, Light Sources, and Application Parameters

\begin{tabular}{|c|c|c|c|c|}
\hline & \multicolumn{4}{|c|}{ Treatment units (groups 1 - 4) } \\
\hline & ELT 2 & ELT 30 & C 46 sun & $C V T / R V T$ \\
\hline Technology & Energizing light (ELT) & Energizing light (ELT) & Red light (RLT) & Red light (RLT) \\
\hline Lamp type & Medium pressure & Medium pressure & Low pressure & Low pressure \\
\hline Treatment area & Partial-body & Full-body & Full-body & Full-body \\
\hline Treatment position & Semi-reclined & Horizontal & Horizontal & Vertical \\
\hline Irradiance $(611-650 \mathrm{~nm})$ & $7.1 \mathrm{~mW} / \mathrm{cm}^{2}$ & $10.4 \mathrm{~mW} / \mathrm{cm}^{2}$ & $5.9 \mathrm{~mW} / \mathrm{cm}^{2}$ & $13.3 \mathrm{~mW} / \mathrm{cm}^{2}$ \\
\hline Total irradiance $(570-850 \mathrm{~nm})$ & $42.8 \mathrm{~mW} / \mathrm{cm}^{2}$ & $54.8 \mathrm{~mW} / \mathrm{cm}^{2}$ & $10.3 \mathrm{~mW} / \mathrm{cm}^{2}$ & $23.4 \mathrm{~mW} / \mathrm{cm}^{2}$ \\
\hline Treatment duration & $20 \min$ & $15 \min$ & $25 \min$ & $12 \mathrm{~min}$ \\
\hline Treatment dose (611-650 nm) & $8.5 \mathrm{~J} / \mathrm{cm}^{2}$ & $9.4 \mathrm{~J} / \mathrm{cm}^{2}$ & $8.9 \mathrm{~J} / \mathrm{cm}^{2}$ & $9.6 \mathrm{~J} / \mathrm{cm}^{2}$ \\
\hline Total radiant exposure $(570-850 \mathrm{~nm})$ & $51.4 \mathrm{~J} / \mathrm{cm}^{2}$ & $49.3 \mathrm{~J} / \mathrm{cm}^{2}$ & $15.5 \mathrm{~J} / \mathrm{cm}^{2}$ & $16.8 \mathrm{~J} / \mathrm{cm}^{2}$ \\
\hline
\end{tabular}

subjective and clinical parameters was conducted at $\mathrm{t} 30+6$ months.

\section{Light Sources}

Four units equipped with two different types of polychromatic light sources (low-pressure vs. mid-pressure lamps) were used to conduct this study. Table 2 lists the lamp technologies, lamp types, treatment area (full or part of the body), spectral values, session duration, and treatment doses for the units used in this study.

Treatment units 2, 3, and 4 provided full-body irradiation, covering the ventral and dorsal surfaces of the head, neck, trunk, upper limbs, and lower limbs at the same time. Fullbody irradiation units 2 and 3 enabled treatment with the patient in a horizontal, reclined position, whereas unit 4 was engineered as a cabin for vertical treatment orientation. Unit 1 was designed for the local treatment of the face and décolletage area with the patient sitting in a chair in a semi-reclined position. Units 1 and 2 were equipped with medium-pressure gas discharge lamps in combination with spectrally selective reflectors and corresponding filter systems, to eliminate spectral emissions in wavelengths $<570$ and $>850 \mathrm{~nm}$; these units were denoted as ELT. Units 3 and 4 were equipped with lowpressure gas discharge fluorescent lamp tubes providing a spectral emission peak predominantly within the range of 611$650 \mathrm{~nm}$, denoted as RLT. Because of the different spectral properties and irradiances, we defined the spectral range between 611 and $650 \mathrm{~nm}$ for the calculation of treatment fluences. This wavelength window encompasses $632.8 \mathrm{~nm}$, which is a paramount wavelength in LLLT and PBM, representing the dominant wavelength of a HeNe-laser. The spectral dose distributions of the ELT and RLT light sources are shown in Fig. 1, with the doses of both light sources normalized to $100 \%$ for the $611-650 \mathrm{~nm}$ range. The treatment doses were kept constant for this spectral range, whereas irradiances and treatment durations varied for all four treatment groups in order to investigate the applicability of the Bunsen-Roscoe law of reciprocity within the given parametrical limits.

All units emitted almost no erythemogenic UV radiation (minimal erythema dose would not be reached after several hours of exposure, comparable to the UV emission of fluorescent lamps for general lighting service applications).

\section{Measurements}

The primary objective of the study was the improvement of subjective skin complexion and skin feeling. The volun- teers were asked to specify their level of agreement to the statements in the questionnaire by marking a position along a continuous black line between two end points measuring $10 \mathrm{~cm}$, which served as a visual analog scale (VAS). The secondary objectives were the improvement of measurement parameters using a DermaLab Combo (Cortex Technology, Hadsund, Denmark), a computer-supported skin diagnostics system equipped with a rotating high-resolution ultrasound sensor probe $(20 \mathrm{MHz})$ for the determination of changes in intradermal collagen density, measured as a collagen intensity score (CIS). A Primos ${ }^{\text {lite }}$ digital fringe projection system (GFM Messtechnik, Berlin, Germany) was used to measure the objective arithmetical roughness $\left(R_{a}\right)$ of the skin surface in the periorbital region.

\section{Photography}

The digital photographs for the blinded wrinkle assessment were taken using a Nikon D5100 camera equipped with a Nikkor AF $50 \mathrm{~mm}$ 1:1.4 lens (Nikon Corporation, Chiyoda, Tokyo, Japan) and a Walimex RFL-3 ring light (Walser GmbH \& Co. KG, Burgheim, Germany).

\section{Subject outcome assessment}

The subjective efficacy parameters were self-assessed at the baseline (t0), after $15(\mathrm{t} 15)$ and 30 (t30) treatments, and

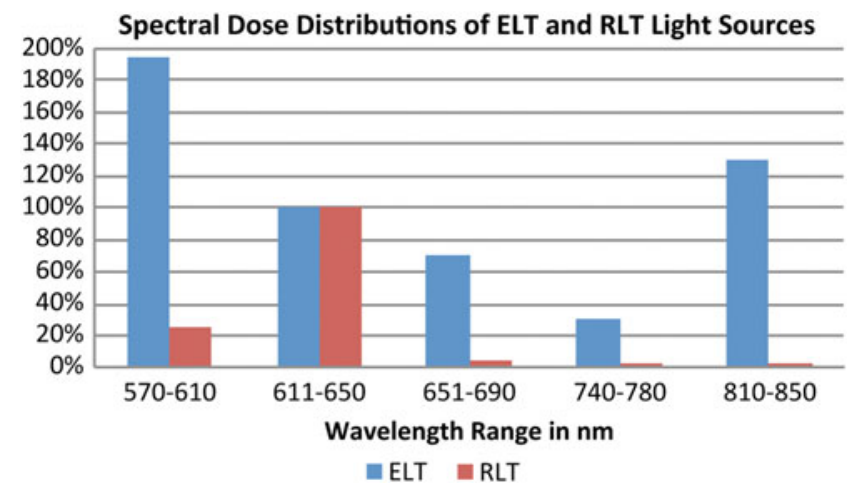

FIG. 1. Spectral dose distributions of energizing light technology (ELT) and red light technology (RLT) light sources. Relationship between doses and wavelength ranges for ELT and RLT light sources, normalized to the spectral range $611-650 \mathrm{~nm}$. Colored bars represent the spectral doses in percentages. 
after $\mathrm{t} 30+6$ months using $10 \mathrm{~cm}$ VAS for the improvements in skin complexion and skin feeling. These parameters were not assessed in the control group.

\section{Objective clinical parameter assessment}

The high-resolution ultrasound examination of collagen has enabled the measurement of visible changes in collagen density and numerical CISs representing the intradermal collagen fiber density. Profilometry yielded a numerical value for the $R_{a}$ of the skin area under examination.

\section{Investigator assessment}

Three independent physicians who were blinded to the clinical patient data, analyzed the clinical photographs obtained at $\mathrm{t} 0$ and $\mathrm{t} 30$. The investigators were instructed to arrange the randomly assorted sets of clinical photographs taken at $\mathrm{t} 0$ and $\mathrm{t} 30$ into a before/after treatment sequence. The baseline wrinkle depth according to the Modified Fitzpatrick Wrinkle Scale (MFWS) ${ }^{12}$ and the degree of wrinkle reduction after treatment had to be assessed after sequencing. The votes of the investigators were summarized by the following majority rules: if two or three experts voted the same way, the agreed-upon classification was the summary measure; if all three experts voted differently, "no change" was the summary measure.

\section{Statistical methods}

The data in the tables are given as means \pm standard deviations. Comparisons of the changes in skin feeling, skin complexion, roughness, and collagen intensity from the baseline to $\mathrm{t} 30$ between the different treatment groups (intergroup comparisons) were performed using a linear model, with the baseline value of each volunteer as a covariate. Within-group differences from the baseline to values at t30 were assessed using the Mann-Whitney-Wilcoxon test. To compare wrinkle difference assessments among groups, we used the $\chi^{2}$ test. Within groups, we tested the hypothesis of equal probabilities of improvement and worsening using binomial tests. All tests were two sided, and $p$ values $<0.05$ were considered statistically significant.

\section{Results}

\section{Patient characteristics}

Initially, 144 volunteers were recruited for the trial. Eight volunteers did not appear for the first appointment after randomization; therefore, the total number of patients finally included in the study was 136. Five volunteers stopped participating because of schedule incompatibilities and lack of time. One volunteer could not finish the treatment because of receiving antibiotic medication, which was one of the exclusion criteria; one volunteer terminated participation because of moving away; and one participant missed more than four treatments because of a period of residence at a health resort. Ultimately, 128 volunteers completed the treatment and the follow-up evaluation course, of whom 57 were treated with RLT, 48 were treated with ELT, and 23 were controls. The volunteers in the RLT and ELT groups were similar with respect to age, weight, skin complexion, skin feeling, skin roughness, and intradermal collagen density. The percentage of women was lower in the ELT group than in the RLT group. The controls had a slightly higher mean collagen density and a lower mean skin roughness.

\section{Adverse events}

None of the volunteers dropped out because of an adverse event. No severe adverse events were registered during the study or the follow-up phase. One volunteer with facial telangiectasia noticed an increased visibility after the first treatments, and decided to protect the zones in question from the light influence using a concealer for the rest of the treatment series. One volunteer experienced a reddening of scar tissue from a 40-year-old knee injury that was likely reactivated by the ELT 30 treatment. The affected scar healed completely within 1 week, and the treatments were continued without interruption.

\section{Assessment of effects}

Figure 2 shows two series of collagen ultrasonography scans, demonstrating the collagen density increase from to to $\mathrm{t} 30$ for one subject each in the RLT group and the ELT group.

Clinical photography revealed visible changes in wrinkles and skin roughness. Figure 3 shows an example for one
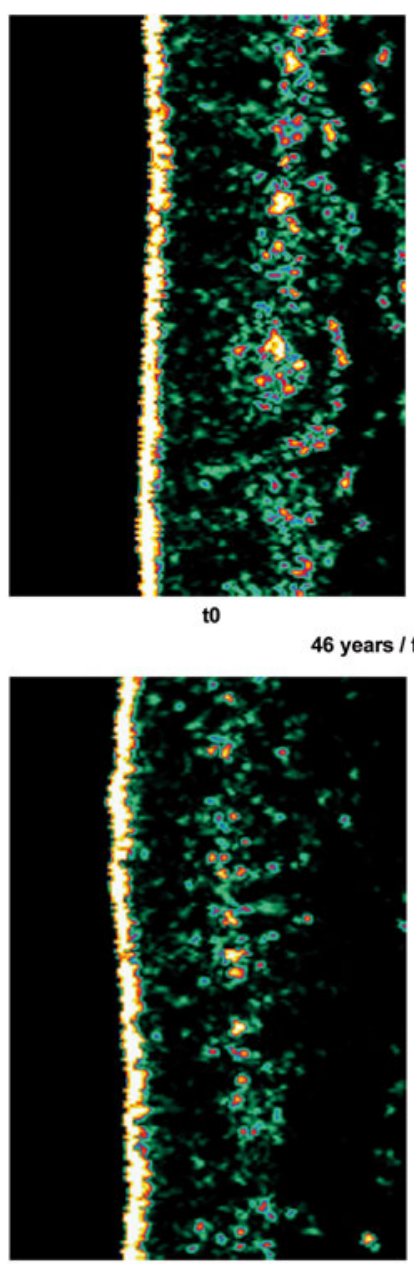

46 years / female / RLT
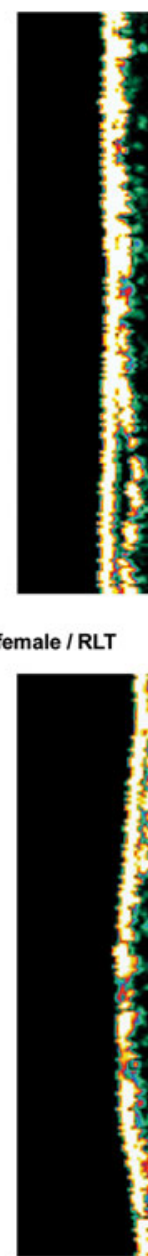

years / female / ELT

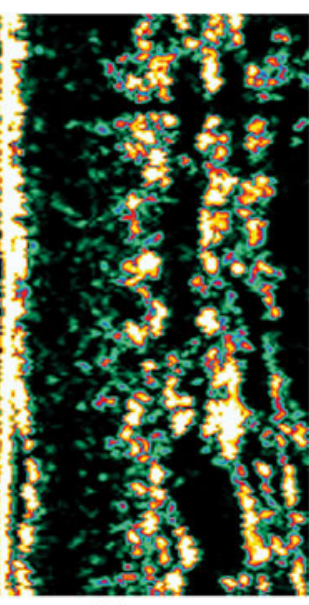

t30

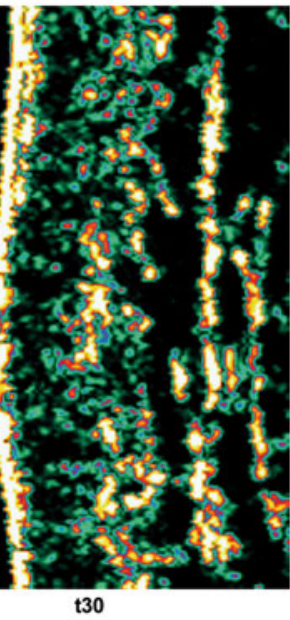

$\mathbf{t} 30$
FIG. 2. Collagen ultrasonography examples. 


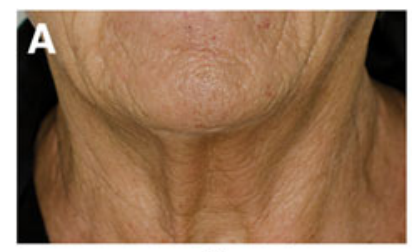

to

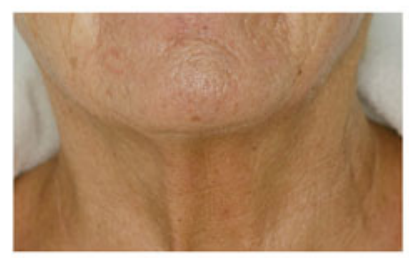

$\mathbf{t 3 0}$

64 years / female / ELT

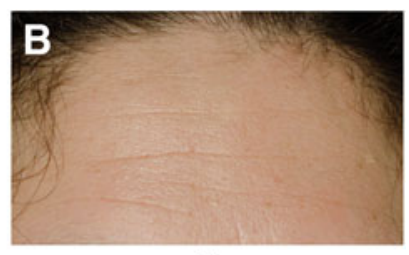

to

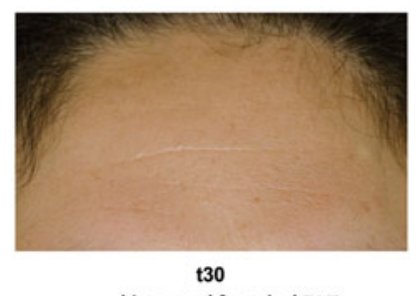

41 years / female / RLT
FIG. 3. Patient photography examples. (A) 64-year-old woman, energizing light technology (ELT). (B) 41-year-old woman, red light technology (RLT).

subject in each treatment group, comparing the baseline ( $\mathrm{t} 0$ ) status with $\mathrm{t} 30$.

In Table 3 , the results of the $\mathrm{t} 30-\mathrm{t} 0$ measurements for each parameter in the different patient groups and the results of the expert wrinkle assessment are summarized. Withingroup comparisons addressed whether the $\mathrm{t} 30-\mathrm{t} 0$ differences had means of zero for each patient group separately.

Within-group comparisons, $\mathrm{t30}$ - t0. In the RLT and ELT groups, skin complexion, skin feeling, collagen intensity score, skin roughness, and wrinkle status improved significantly ( $p<0.001$, Table 3$)$. The skin feeling, skin complexion, and roughness changes were significantly $(p<0.001$, covariance analysis) correlated with baseline values in all groups. In contrast, the control subjects showed no significant difference in collagen density and significant worsening of skin roughness and wrinkle status. These results are described in greater detail in Fig. 4. Here, baseline measurements on the $\mathrm{x}$-axis and the respective gain or reduction in the $\mathrm{t} 30$ values on the $y$-axis are color coded for the different treatment groups. In Fig. 4A, B and D, nearly all of the ELT and RLT points plotted below the baseline $x$-axis $=0.00$, indicating that the skin feeling, skin complexion, and roughness improved for nearly all of the volunteers $(p<0.01)$. In Fig. 4C (CIS), the baseline effect is not significant, whereas the CIS increase is significant $(p<0.001)$, and values above the $\mathrm{x}$-axis indicate improvement.

Between-group comparisons. For the main efficacy parameters, skin complexion and skin feeling, we observed no significant differences between the RLT and ELT groups. The collagen density, roughness, and wrinkle status were significantly different among the three groups, as shown in Table 3. There was no difference between the RLT and ELT groups, but there was a difference between both groups compared with controls, as shown by the blue points in Fig. $4 \mathrm{C}$ and D.

Subgroup analyses. We wanted to assess whether the two RLT treatment groups and the two ELT treatment groups showed different results; therefore, we compared the two groups. The RLT subgroups had 25 volunteers using CVT/RVT and 32 using C46 sun. There were no differences between the two groups with respect to skin complexion, skin feeling, skin roughness, collagen density, and wrinkle status. All of these parameters improved significantly between $\mathrm{t} 0$ and $\mathrm{t} 30$ (data not shown). We obtained very similar results for the two ELT groups, with 27 volunteers in ELT 30 and 21 volunteers in ELT 2.

The RLT group consisted of a lower percentage of male volunteers than did the ELT group and the control. Gender differences regarding the response to the PBM treatment for the main parameters were tested within each of the RLT/ ELT/control subgroups using the Mann-Whitney $U$ test, and we found no significant differences $(p>0.1$ for all tests). Including gender as an additional covariate in the covariance analysis resulted in very similar $p$ values for the tests regarding the comparison of study groups, compared with the analysis without gender. Only for collagen increase were gender and treatment both significant.

\section{Long-term follow-up}

The long-term results were analyzed for all subjects who were available for long-term follow-up in November/ December 2012. A total of 52 of the 77 subjects who took part in the long-term follow-up finished after 30 treatments, 18

Table 3. Comparison of the $\mathrm{t} 30$ - to Results Between and Within Subject Groups

\begin{tabular}{|c|c|c|c|c|c|c|c|}
\hline & $R I T(\mathrm{n}=57)$ & Within-group & FIT $(\mathrm{n}=48)$ & Within-group & Controls & Within-group & Between- \\
\hline & & & & & & & \\
\hline Skin complexion (subjective) ${ }^{a}$ & $-1.29 \pm 1.98$ & $<0.001$ & $-1.72 \pm 2.35$ & $<0.001$ & & & 0.064 \\
\hline Skin feeling (subjective) ${ }^{\mathrm{a}}$ & $-1.01 \pm 2.30$ & $<0.001$ & $-1.65 \pm 2.17$ & $<0.001$ & & & 0.167 \\
\hline Skin roughness $\left(R_{a}\right)^{a}$ & $-1.79 \pm 2.46$ & $<0.001$ & $-1.58 \pm 2.22$ & $<0.001$ & $0.95 \pm 1.45$ & 0.003 & 0.003 \\
\hline Collagen intensity score ${ }^{\mathrm{b}}$ & $5.75 \pm 4.54$ & $<0.001$ & $6.40 \pm 5.17$ & $<0.001$ & $-0.26 \pm 5.09$ & 0.84 & $<0.001$ \\
\hline Expert wrinkle assessment ${ }^{\mathrm{C}}$ & & $<0.001$ & & $<0.001$ & & $<0.001$ & $<0.001$ \\
\hline Better & $40 / 69 \%$ & & $36 / 75 \%$ & & $1 / 4 \%$ & & \\
\hline Equal & $8 / 14 \%$ & & $7 / 15 \%$ & & $5 / 22 \%$ & & \\
\hline Worse & $10 / 17 \%$ & & $5 / 10 \%$ & & $17 / 74 \%$ & & \\
\hline
\end{tabular}

${ }^{a}$ Values represent means $\pm S D$ of the difference $\mathrm{t} 30-\mathrm{t} 0$; negative numbers indicate improvement.

${ }^{b}$ Values represent means $\pm S D$ of the difference $\mathrm{t} 30-\mathrm{t} 0$; positive numbers indicate improvement.

${ }^{\mathrm{c}}$ Majority vote of three blinded expert reviewers, $\chi^{2}$ test for comparisons between groups, binomial test for within-group comparisons.

Analysis of covariance for the between-group comparison, one sample Wilcoxon-test for the within-group comparisons.

RLT, red light technology; ELT, energizing light technology. 


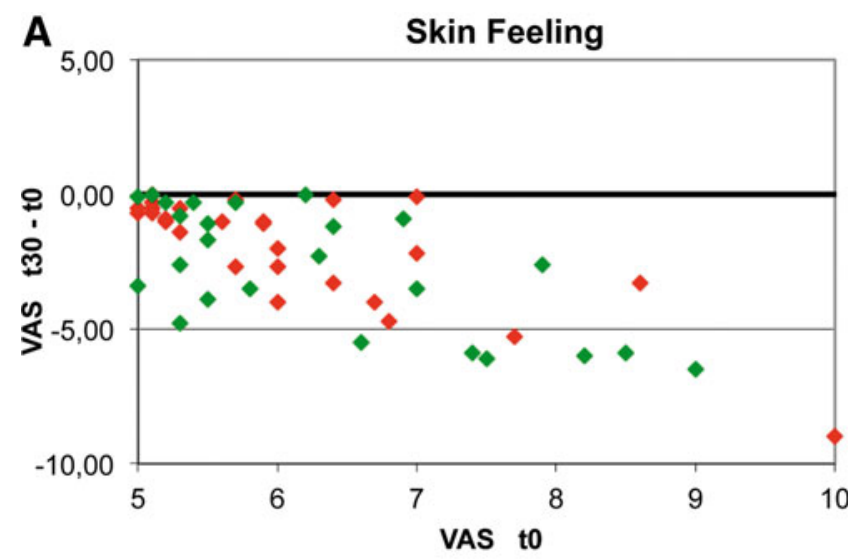

RLT

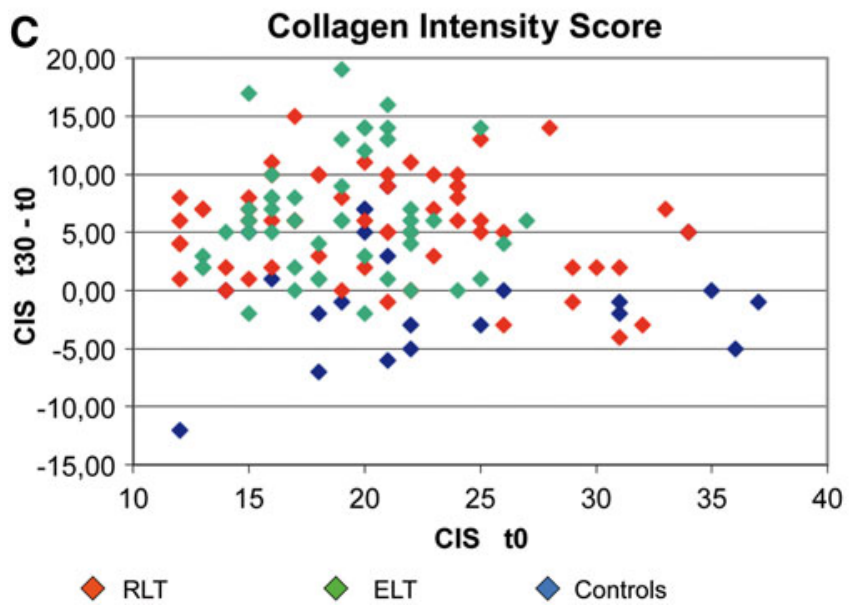

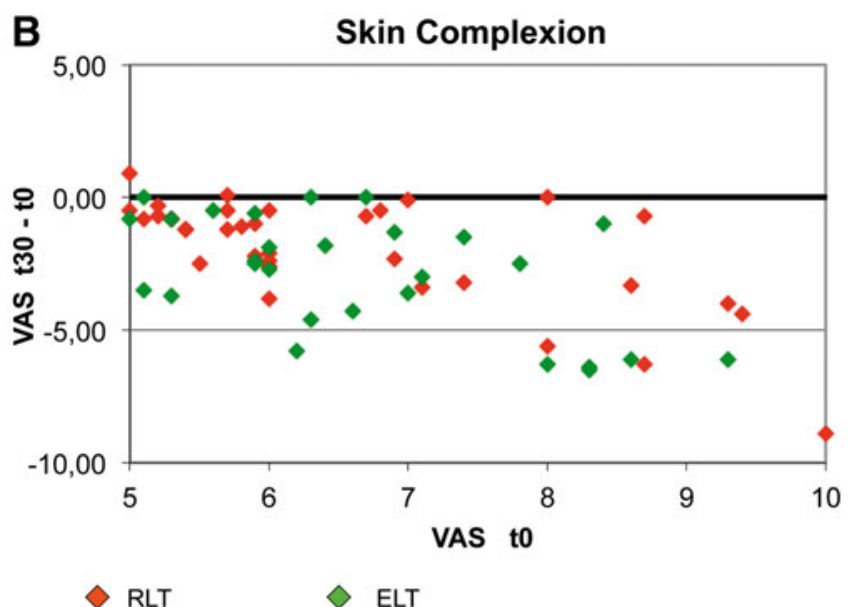

D

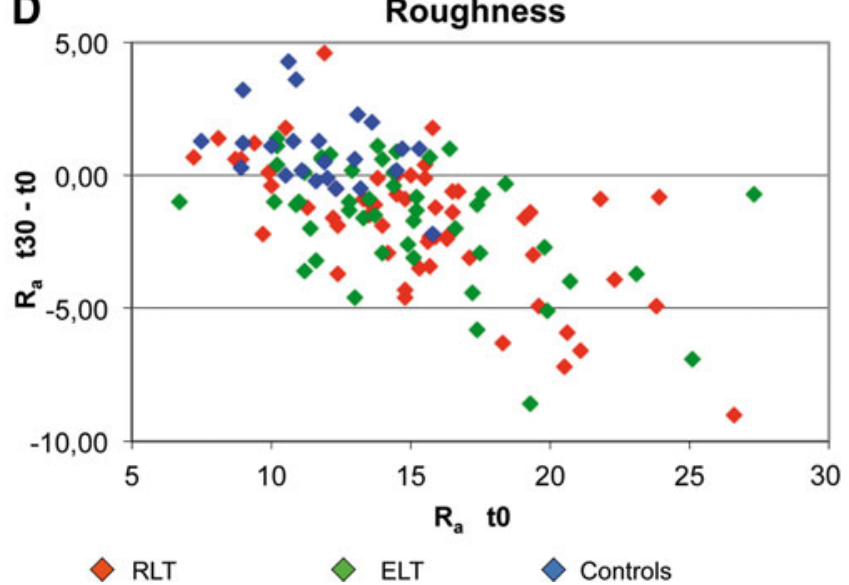

FIG. 4. Results for $\mathrm{t} 30-\mathrm{t} 0$. Changes $\mathrm{t} 30-\mathrm{t} 0$ (y-axis) are depicted in relation to the baseline value $\mathrm{t} 0$ on the $\mathrm{x}$-axis. For $\mathbf{A}$, B, and $\mathbf{D}$, points below the $x$-axis indicate improvement; for $\mathbf{C}$, points above the $x$-axis indicate improvement. The red light technology (RLT) and energizing light technology (ELT) t30 - t0 differences decrease with increasing baseline values.

volunteers continued to a total of 45 treatments, and 7 volunteers received a total of 60 treatments $(\mathrm{t} 60)$. To analyze the long-term effects, we tested whether the t60 measurements of skin feeling, skin complexion, CIS, and $\mathrm{R}_{\mathrm{a}}$ were better than the t0 measurements for the group of volunteers with 30 treatments. All volunteers had significantly better results at t60 (Wilcoxon test $\leq 0.001$ for each). The $\mathrm{t} 60-\mathrm{t} 0$ differences were as follows: mean 0.99, SD 1.95 for skin feeling; mean -1.00 , SD 2.10 for skin complexion; mean 5.10, SD 7.56 for CIS; and mean -0.64, SD 3.53 for $\mathrm{R}_{\mathrm{a}}$. As expected, these differences displayed lower effect sizes than at $\mathrm{t} 30$. Only a group of seven volunteers continued the therapy with good results for a further 30 treatments, which may be partly the result of selection bias. Therefore, the long-term efficacy must be systematically evaluated in further studies. During the follow-up period, no delayed adverse events were recorded.

\section{Discussion}

The use of LED light sources with 590, 633, and $830 \mathrm{~nm}$ wavelengths for athermal light-only photorejuvenation has grown rapidly in recent years. Additional wavelengths have been shown to be efficient in altering cellular functions, such as $570,{ }^{13} 620,680,760$, and $820 \mathrm{~nm} .^{14}$ The treatment doses vary significantly, ranging from $0.1 \mathrm{~J} / \mathrm{cm}^{2}$ for $590 \mathrm{~nm}$ LED light with a specific sequence of pulsing, ${ }^{15}$ up to $126 \mathrm{~J} /$ $\mathrm{cm}^{2}$ for $633 \mathrm{~nm}$ continuous LED light. ${ }^{16,17}$ The power of the light typically ranges between 1 and $1000 \mathrm{~mW}$, depending upon the type of light source and the application. ${ }^{1}$ Efficacy comparisons of the different devices available to the physician are not known to the authors.

This study is the first prospective clinical trial investigating the safety and efficacy of novel light sources for skin rejuvenation and the stimulation of dermal collagen synthesis based on low-pressure and mid-pressure gas discharge lamps. These light sources, in contrast to lasers and LEDs, allow simultaneous treatment with a tailored spectrum composed of several spectral bands that are effective in PBM. When compared with the initial values and the controls, the volunteers experienced significant improvements in their personal assessments of skin feeling and complexion, in clinical outcomes as assessed by collagen density and skin roughness measurements and in the reduction of fine lines and wrinkles as assessed by three blinded evaluators comparing $\mathrm{t} 0$ and $\mathrm{t} 30$ photographs.

Previous findings were able to correlate fibroblast activity and dermal matrix remodeling processes, with an increase in 
intradermal collagen density and reduced signs of aging. ${ }^{18}$ The proposed underlying mechanisms include the photostimulation of terminal molecules in the electron transport chain and the subsequent adenosine triphosphate (ATP) concentration increase, ${ }^{14}$ along with the selective light-driven activation of water molecules, ${ }^{19}$ thereby enhancing metabolic exchange and influencing the ion transporter systems found in cellular membranes. ${ }^{20}$ Detailed analysis of the gene expression profiles in human fibroblasts revealed an influence of low-intensity red light with a 628-nm wavelength on 111 different genes that are involved in cellular functions, such as cell proliferation; apoptosis; stress response; protein, lipid and carbohydrate metabolism; mitochondrial energy metabolism; DNA synthesis and repair; antioxidant related functions; and cytoskeleton- and cell-cell interaction-related functions. ${ }^{21}$ A specific role of reactive oxygen species (ROS) in increasing fibroblast proliferation and motility has recently been reported, suggesting that the elevation of ROS via photodynamic therapy can enhance the cellular functions of dermal fibroblasts through specific mitogen-activated protein kinase (MAPK) signaling pathways in vitro. ${ }^{22}$ The light-induced free radical formation in human skin has been investigated in detail, demonstrating that red light with 620 and $670 \mathrm{~nm}$ wavelengths increases the concentration of ROS even without the influence of external photosensitizers. ${ }^{23}$

Because fibroblasts are responsible for collagen production in wound healing, dermal remodeling, and tissue repair, we decided to focus on increased collagen density as a surrogate marker for fibroblast activity, and abandoned such invasive monitoring methods as histologic examinations following skin biopsies for our study. Ultrasonographic collagen assessment is described as a feasible noninvasive methodology for monitoring dermal density during the senescence process. $^{24}$

A report of the stimulatory effects of $660 \mathrm{~nm}$ wavelength laser light on scar fibroblasts ${ }^{25}$ could conceivably explain the potential reactivation of a $>40$-year-old knee injury, which occurred in one volunteer during the ELT treatment. Therefore, the influence of PBM on scar tissue should be subject to further investigation.

Some authors emphasize the importance of distinct wavelengths for optimal results. ${ }^{16-18,26-28}$ In our study, the differences between the RLT and ELT treatments in clinical outcome and patient satisfaction were not significant, indicating that despite spectral differences, both light sources were commensurably effective regarding study objectives. Further studies of the treatment parameters are necessary.

The evaluation of clinical photography revealed a particular worsening of fine lines and wrinkles from t0 to $t 30$ in the control group, which was not expected for a course of only 12 weeks. A possible explanation could be the seasonal variation of skin condition between winter and summer climates and the influence of solar radiation, as the clinical photography revealed skin pigmentation as a consequence of exposure to sunlight.

We observed a tendency that ELT/RLT treatment led to better results in female volunteers regarding the collagen density increase. This gender-specific response could conceivably be explained by physiological differences between male and female skin ${ }^{29,30}$ on endocrine and extracellular matrix levels. However, gender-specific differences should be evaluated in greater detail in further investigations.

\section{Conclusions}

RLT and ELT are large-area and full-body treatment modalities for skin rejuvenation and improvements in skin feeling and skin complexion. The application of RLT and ELT provides a safe, non-ablative, non-thermal, atraumatic photobiomodulation treatment of skin tissue with high patient satisfaction rates. RLT and ELT can extend the spectrum of anti-aging treatment options available to patients looking for mild and pleasant light-only skin rejuvenation.

\section{Acknowledgments}

We thank Dr. Christine Fischer, Heidelberg, for help and advice regarding the statistical analysis of our data. We also thank all of the volunteers for their participation in this study. This study was fully funded by JK-Holding $\mathrm{GmbH}$, Windhagen, Germany. All materials, light sources, and evaluation equipment were provided by the sponsor.

\section{Author Disclosure Statement}

The principal investigator (Alexander Wunsch) was mandated and remunerated by the sponsor to conduct the study. The authors have received funds to plan, conduct, and evaluate the study.

\section{References}

1. Chung H., Dai T., Sharma S., Huang Y.Y., Carroll J., and Hamblin M. (2012). The nuts and bolts of low-level laser (light) therapy. Ann. Biomed. Eng. 40, 516-533.

2. Anderson R.R., and Parrish J.A. (1981). The optics of human skin. J. Invest. Dermatol. 77, 13-19.

3. Gupta A.K., Filonenko N., Salansky N., and Sauder D.N. (1998). The use of low energy photon therapy (LEPT) in venous leg ulcers: a double-blind, placebo-controlled study. Dermatol. Surg. 24, 1383-1386.

4. Minatel D.G., Frade M.A., Franca S.C., and Enwemeka C.S. (2009). Phototherapy promotes healing of chronic diabetic leg ulcers that failed to respond to other therapies. Lasers Surg. Med. 41, 433-441.

5. Barolet D., Roberge C.J., Auger F.A., Boucher A., and Germain L. (2009). Regulation of skin collagen metabolism in vitro using a pulsed $660 \mathrm{~nm}$ LED light source: clinical correlation with a single-blinded study. J. Invest. Dermatol. 129, 2751-2759.

6. Huang, Y.Y., Chen, A.C.H., Carroll, J.D., and Hamblin, M.R. (2009). Biphasic dose response in low level lightherapy. Dose Response 7, 358-383.

7. Calderhead R.G. (2007). The photobiological basics behind light-emitting diode (LED) phototherapy. Laser Ther. 16, 97108.

8. Papadavid E., and Katsambas A. (2003). Lasers for facial rejuvenation: A review. Int. J. Dermatol. 42, 480-487.

9. Khoury J.G., and Goldman M.P. (2008). Use of light-emitting diode photomodulation to reduce erythema and discomfort after intense pulsed light treatment of photodamage. J. Cosmet. Dermatol. 7, 30-34.

10. Smith K.C. (2005). Laser (and LED) therapy is phototherapy. Photomed. Laser Surg. 23, 78-80.

11. van Breugel H.H., and Bär P.R. (1992). Power density and exposure time of $\mathrm{He}-\mathrm{Ne}$ laser irradiation are more important than total energy dose in photo-biomodulation of human fibroblasts in vitro. Lasers Surg. Med. 12, 528-537.

12. Shoshani D., Markovitz E., Monsterey S.J., and Narins D.J. (2008). The Modified Fitzpatrick Wrinkle Scale: A clinical 
validated measurement tool for nasolabial wrinkle severity assessment. Dermatol. Surg. 34, 85-91.

13. Vinck E.M., Cagnie B.J., Cornelissen M.J., Declercq H.A., and Cambier D.C. (2005). Green light emitting diode irradiation enhances fibroblast growth impaired by high glucose level. Photomed. Laser Surg. 23, 167-171.

14. Karu T.I. (2010). Multiple roles of cytochrome c oxidase in mammalian cells under action of red and IR-A radiation. IUBMB Life 62, 607-610.

15. Weiss R.A., McDaniel D.H., Geronemus R.G., and Weiss M.A. (2005). Clinical trial of a novel non-thermal LED array for reversal of photoaging: clinical, histologic, and surface profilometric results. Lasers Surg. Med. 36, 85-91.

16. Russell B.A., Kellett N., and Reilly L.R. (2005). A study to determine the efficacy of combination LED light therapy $(633 \mathrm{~nm}$ and $830 \mathrm{~nm})$ in facial skin rejuvenation. J. Cosmet. Laser Ther. 7, 196-200.

17. Sadick N.S. (2008). A study to determine the efficacy of a novel handheld light-emitting diode device in the treatment of photoaged skin. J. Cosmet. Dermatol. 7, 263-267.

18. Lee S.Y., Park K.H., Choi J.W., et al. (2007). A prospective, randomized, placebo-controlled, double-blinded, and splitface clinical study on LED phototherapy for skin rejuvenation: Clinical, profilometric, histologic, ultrastructural, and biochemical evaluations and comparison of three different treatment settings. J. Photochem. Photobiol. B. 88, 51-67.

19. Santana-Blank L., Rodríguez-Santana E., and SantanaRodríguez K.E. (2012). Photobiomodulation of aqueous interfaces as selective rechargeable bio-batteries in complex diseases: personal view. Photomed. Laser Surg. 30, 242-249.

20. Calderhead R.G., Kubota J., Trelles M.A., and Ohshiro T. (2008). One mechanism behind LED phototherapy for wound healing and skin rejuvenation: Key role of the mast cell. Laser Therapy 17, 141-148.

21. Zhang Y., Song S., Fong C.C., et al. (2003). cDNA microarray analysis of gene expression profiles in human fibroblast cells irradiated with red light. J. Invest. Dermatol. 120, 849-857.

22. Jang Y.H., Koo G.B., Kim J.Y., Kim Y.S., and Kim Y.C. (2013). Prolonged activation of ERK contributes to the photo- rejuvenation effect in photodynamic therapy in human dermal fibroblasts. J. Invest. Dermatol. 133, 2265-2275.

23. Zastrow L., Groth N., Klein F., et al. (2009). The missing link-light-induced $(280-1,600 \mathrm{~nm})$ free radical formation in human skin. Skin Pharmacol. Physiol. 22, 31-44.

24. Crisan D., Crisan M., Moldovan M., Lupsor M., and Badea R. (2012). Ultrasonographic assessment of the cutaneous changes induced by topical flavonoid therapy. Clin. Cosmet. Investig. Dermatol. 5, 7-13.

25. Webb C., Dyson M., and Lewis W.H. (1998). Stimulatory effect of $660 \mathrm{~nm}$ low level laser energy on hypertrophic scarderived fibroblasts: possible mechanisms for increase in cell counts. Lasers Surg. Med. 22, 294-301.

26. Baez F., and Reilly L.R. (2007). The use of light-emitting diode therapy in the treatment of photoaged skin. J. Cosmet. Dermatol. 6, 189-194.

27. Vinck E.M., Cagnie B.J., Cornelissen M.J., Declercq H.A., and Cambier D.C. (2003). Increased fibroblast proliferation induced by light emitting diode and low power laser irradiation. Lasers Med. Sci. 18, 95-99.

28. Goldberg D.J., Amin S., Russell B.A., Phelps R., Kellett N., and Reilly L.A. (2006). Combined 633-nm and 830-nm led treatment of photoaging skin. J. Drugs Dermatol. 5, 748-753.

29. Giacomoni P.U., Mammone T., and Teri M. (2010). Genderlinked differences in human skin. J. Dermatol. Sci. 55, 144149.

30. Oh, J.H., Kim Y.K., Jung J.Y., et al. (2011). Intrinsic agingand photoaging-dependent level changes of glycosaminoglycans and their correlation with water content in human skin. J. Dermatol. Sci. 62, 192-201.

Address correspondence to: Alexander Wunsch Hirschgasse 11 69120 Heidelberg Germany

E-mail: praxis@alexanderwunsch.de 